\title{
Determinants of the omega-3 index in a Mediterranean population at increased risk for CHD
}

\author{
Aleix Sala-Vila ${ }^{1,2 *}$, William S. Harris ${ }^{3}$, Montserrat Cofán ${ }^{1,2}$, Ana M. Pérez-Heras ${ }^{1,2}$, Xavier Pintó ${ }^{4,5}$, \\ Rosa M. Lamuela-Raventós ${ }^{5,6}$, Maria-Isabel Covas ${ }^{7}$, Ramon Estruch ${ }^{2,8}$ and Emilio Ros ${ }^{1,2}$ \\ ${ }^{1}$ Lipid Clinic, Endocrinology and Nutrition Service, Institut d'Investigacions Biomèdiques August Pi Sunyer (IDIBAPS), \\ Unitat de Lipids, Hospital Clinic, Villarroel 170, 08036 Barcelona, Spain \\ ${ }^{2}$ Ciber Fisiopatología de la Obesidad y Nutrición (CIBERobn), Instituto de Salud Carlos III (ISCIII), Malaga, Spain \\ ${ }^{3}$ Cardiovascular Health Research Center, Sanford Research/USD, Sioux Falls, SD, USA \\ ${ }^{4}$ Atherosclerosis Unit, Department of Internal Medicine, IDIBELL-Hospital Universitari de Bellvitge, L'Hospitalet de Llobregat, \\ Barcelona, Spain \\ ${ }^{5}$ Redes Temáticas de Investigación Cooperativa (RETIC) RD06/0045, ISCIII, Spain \\ ${ }^{6}$ Nutrition and Food Science Department, XaRTA, INSA, Pharmacy School, University of Barcelona, Barcelona, Spain \\ ${ }^{7}$ Cardiovascular Risk and Nutrition Research Group, Institut Municipal d'Investigació Mèdica (IMIM), Barcelona, Spain \\ ${ }^{8}$ Department of Internal Medicine, IDIBAPS, Hospital Clinic, Barcelona, Spain
}

(Received 9 September 2010 - Revised 4 January 2011 - Accepted 6 January 2011 - First published online 30 March 2011)

\section{Abstract}

The omega- 3 index, defined as the sum of EPA and DHA in erythrocyte membranes expressed as a percentage of total fatty acids, has been proposed as both a risk marker and risk factor for CHD death. A major determinant of the omega-3 index is EPA + DHA intake, but the impact of other dietary fatty acids has not been investigated. In a cross-sectional study on 198 subjects (102 men and 96 women, mean age 66 years) at high cardiovascular risk living in Spain, the country with low rates of cardiac death despite a high prevalence of cardiovascular risk factors, dietary data were acquired from FFQ and blood cell membrane fatty acid composition was measured by GC. The average consumption of EPA + DHA was $0.9 \mathrm{~g} / \mathrm{d}$ and the mean omega-3 index was $7 \cdot 1 \%$. In multivariate models, EPA + DHA intake was the main predictor of the omega-3 index but explained only $12 \%$ of its variability $(P<0 \cdot 001)$. No associations with other dietary fatty acids were observed. Although the single most influential determinant of the omega- 3 index measured here was the intake of EPA + DHA, it explained little of the former's variability; hence, the effects of other factors (genetic, dietary and lifestyle) remain to be determined. Nevertheless, the high omega-3 index could at least partially explain the paradox of low rates of fatal CHD in Spain despite a high background prevalence of cardiovascular risk factors.

Key words: Diet: Cardiovascular risk: EPA: DHA: PUFA: SFA

There is a large body of evidence on the cardiovascular benefits of $n-3$ long-chain PUFA (marine $n-3$ fatty acids), mainly EPA $(20: 5 n-3)$ and DHA $(22: 6 n-3)^{(1)}$. The synthesis of these fatty acids is extremely inefficient in humans ${ }^{(2)}$, and thus they must be acquired pre-formed from dietary sources, mainly from fatty fish. Dietary EPA and DHA are readily incorporated into cell membranes where they influence membrane function, and this is believed to be one of the mechanisms underlying their anti-arrhythmic, lipid-lowering, anti-thrombotic and overall anti-atherosclerotic effects ${ }^{(1)}$. The strength of the evidence favouring $n-3$ fatty acids has prompted many international health organisations and agencies to publish recommendations for increased $n$ - 3 fatty acid intake, typically by advising the inclusion of at least two servings/week of fatty fish to promote cardiovascular health ${ }^{(3)}$.

The blood lipid contents of EPA and DHA have been widely used as biomarkers of intake and as surrogates of their enrichment in cellular membranes ${ }^{(4)}$. In addition, the omega- 3 index, defined as the sum of percentages of EPA + DHA in erythrocyte membranes, has been proposed as a new risk marker and risk factor for CHD, particularly sudden cardiac death $^{(2)}$. Based on previous studies in Western populations, the cardioprotective target level for the omega-3 index has been tentatively set at $8 \%$, while values below $4 \%$ are

Abbreviations: ALA, $\alpha$-linolenic acid; LA, linoleic acid.

*Corresponding author: Dr A. Sala-Vila, fax +34 934537829, email asala@clinic.ub.es 
associated with higher cardiovascular risk ${ }^{(5)}$. The main determinant of the omega-3 index is the consumption of $\mathrm{EPA}+\mathrm{DHA}^{(6-8)}$, which explains the higher values reported in Japanese populations compared with those from the USA or other Western countries ${ }^{(6-9)}$. In recent years, some other factors that influence the omega-3 index have been described, including age, BMI, diabetes, smoking, physical activity and socio-economic status ${ }^{(6-9)}$. The influence of dietary fatty acids other than EPA and DHA on the omega-3 index has been little investigated but is a subject of interest. This is particularly true of the $n-6$ fatty acids linoleic acid (LA) and arachidonic acid since there is some controversy regarding their role in CHD risk ${ }^{(10-13)}$, and of the plant-derived $n$-3 fatty acid $\alpha$-linolenic acid (ALA) because it is a precursor of longchain $n$-3 PUFA. To address these questions, we examined the associations between the omega- 3 index and dietary fatty acids in subjects at high cardiovascular risk living in Spain, the country with the highest fish consumption both in Europe $^{(14)}$ and among Mediterranean countries ${ }^{(15)}$.

\section{Methods}

\section{Subjects}

The present analysis was conducted within the Prevención con Dieta Mediterránea (PREDIMED) study, a large, multicentre, parallel-group, controlled, randomised clinical trial aimed at assessing the effects of the Mediterranean diet on the primary prevention of CVD. PREDIMED has eleven recruitment sites in nine Spanish cities, including one at the Hospital Clinic of Barcelona (Barcelona-North, Spain). The protocol has been reported in detail elsewhere ${ }^{(16)}$. Briefly, participants were men aged between 55 and 80 years and women aged between 60 and 80 years with no prior CVD but at high cardiovascular risk. Inclusion criteria were either type 2 diabetes mellitus or at least three of the following risk factors: current smoking ( $>1$ cigarette/d during the last month); hypertension (systolic blood pressure $\geq 140 \mathrm{mmHg}$ or diastolic blood pressure $\geq 90 \mathrm{mmHg}$ or antihypertensive medication); LDLcholesterol $\geq 1600 \mathrm{mg} / \mathrm{l}$; HDL-cholesterol $\leq 400 \mathrm{mg} / \mathrm{l}$ in men or $\leq 500 \mathrm{mg} / 1$ in women, independently of lipid-lowering therapy; BMI $\geq 25 \mathrm{~kg} / \mathrm{m}^{2}$; family history of premature CHD (definite myocardial infarction or sudden death before 55 years in male first-degree relatives or before 65 years in female first-degree relatives). Exclusion criteria were as follows: previous history of CVD; any severe chronic illness; drug or alcohol addiction; history of allergy or intolerance to olive oil or nuts (supplemental foods given in two arms of the study); low predicted likelihood of changing dietary habits. Between 2007 and 2009, 198 participants were recruited in the Barcelona-North site. At the first visit, participants provided informed consent to participate in the study and have their data about medical history, medication use and lifestyle, including dietary intake to be used. Anthropometric and blood pressure measurements were performed, and fasting blood samples were drawn. The study protocol (ISRCTN 35739639) was conducted according to the guidelines laid down in the Declaration of Helsinki, and all procedures were approved by the ethics committee of the institution. Written informed consent was obtained from all subjects.

\section{Assessment of risk factors}

Participants were considered as diabetic, hyperlipidaemic or hypertensive if they had a previous diagnosis of these conditions and/or they were treated with antidiabetic, cholesterol-lowering or antihypertensive agents, respectively. Smoking status was categorised into never, current or past smoking according to self-reports. Physical activity was determined with the validated Spanish version of the Minnesota questionnaire $^{(17,18)}$. Height, weight and waist circumference were measured with standard methods. Trained personnel measured systolic and diastolic blood pressure in triplicate with a validated semi-automatic oscillometer (Omron HEM-705CP; Omron Healthcare Europe, Hoofddorp, The Netherlands).

\section{Dietary intake}

The dietary habits of participants were assessed using a validated 137 -item $\mathrm{FFQ}^{(19)}$. At the inclusion visit, the FFQ was completed by a trained dietitian in face-to-face interviews. Participants were asked about the frequency of consumption of each food item during the past year, specifying usual portion sizes (semi-quantitative assessment). A total of nine possibilities of frequency were offered, from never to more than six times per $d$. Information on seafood products was collected in eight items of the FFQ (uncanned fatty fish; lean fish; smoked/salted fish; molluscs; shrimp, prawn and crayfish; octopus, baby squid and squid; fatty fish canned in oil; fatty fish canned in salted water). Nutrient intakes were computed using Spanish food composition tables ${ }^{(20)}$ and were adjusted for energy intake by the residual method ${ }^{(21)}$.

\section{Laboratory analyses}

Both fasting serum and $0 \cdot 1 \%$ EDTA blood were collected and processed immediately. Serum lipid and glucose concentrations were determined by standard enzymatic methods in the hospital clinical laboratory. A $100 \mu \mathrm{l}$ aliquot of EDTAcollected blood was transferred into a chloroform-resistant eppendorf containing $1400 \mu \mathrm{l}$ of distilled water. Once cells were haemolysed, they were spun for $5 \mathrm{~min}$ at $4^{\circ} \mathrm{C}$ at $2800 \mathrm{~g}$ in a microcentrifuge (Hermle Z 233 MK-2; Midwest Scientific, St Louis, MO, USA). The supernatant fluid (containing $\mathrm{Hb}$ and serum lipids) was discarded, and the pellet (almost entirely composed of erythrocyte membranes) was extracted with chloroform-methanol $(2: 1, \mathrm{v} / \mathrm{v})$ containing butylated hydroxytoluene $(50 \mathrm{mg} / \mathrm{ml})$ and evaporated to dryness under $\mathrm{N}_{2}$. The lipid extract was stored at $-80^{\circ} \mathrm{C}$ until analysed. The lipid extract was redissolved in $1 \mathrm{ml}$ boron trifluoridemethanol and transferred to a screw-cap test-tube, which was heated for $10 \mathrm{~min}$ at $100^{\circ} \mathrm{C}$ to hydrolyse and methylate the membrane glycerophospholipid fatty acids. The extracts were cooled at $25^{\circ} \mathrm{C}$, and fatty acid methyl esters were isolated 
by adding $300 \mu \mathrm{l}$ of $n$-hexane. After shaking for $1 \mathrm{~min}, 1 \mathrm{ml}$ of a saturated $\mathrm{NaCl}$ solution was added, and the tubes were centrifuged for $10 \mathrm{~min}$ at $2200 \mathrm{~g}$ at room temperature to separate the layers. The upper (hexane) layer was removed, dried with anhydrous sodium sulphate, and a $50 \mu$ l aliquot was transferred into an automatic injector vial equipped with a volume adapter of $300 \mu \mathrm{l}$. Fatty acid methyl esters were separated by GC using a Perkin Elmer Clarus 500 apparatus (Perkin Elmer España, Madrid, Spain) equipped with a $30 \mathrm{~m} \times 0.25$ $\mu \mathrm{m} \times 0.25 \mathrm{~mm}$ SupraWAX-280 capillary column (Teknokroma, Barcelona, Spain), an autosampler and a flame ionisation detector. Each fatty acid is expressed as a percentage of total identified fatty acids in the whole blood sample. The omega-3 index was calculated by the sum of percentages of $\mathrm{EPA}+\mathrm{DHA}$. To ensure the comparability of the omega- 3 indices measured in the laboratory of the Hospital Clinic of Barcelona with that of other centres, ten dried blood samples were analysed, and the results were compared with those obtained in a reference centre (OmegaQuant, LLC, Sioux Falls, SD, USA). The regression coefficient $\left(r^{2}\right)$ between the omega-3 index values was $0.96(P<0.0001)$, and mean values for $\mathrm{EPA}+\mathrm{DHA}$ were $3.84(\mathrm{SD} 2.22) \%$ at OmegaQuant $v .3 .89$ (SD $2 \cdot 11) \%$ in Barcelona.

\section{Statistical analyses}

Univariate regression and one-way ANOVA models were used to determine the effects of each patient's characteristics on the EPA + DHA of whole blood cells. The ANOVA models included independent determinants known to be predictors of the omega-3 index, chosen based on previous literature, i.e. sex, age, being a current smoker, treatment for diabetes, hypertension or dyslipidaemia, physical activity, BMI, and energy-adjusted dietary intakes of total fat and alcohol $(\mathrm{g} / \mathrm{d})^{(6-9,22)}$. Some variables known to be related to the omega-3 index, such as fasting serum TAG and hypertension ${ }^{(7)}$, were left out of the model, as they were considered to be more a consequence than a determinant of the omega- 3 index.

In addition, independent associations between the omega- 3 index and the intake of fatty acids other than EPA + DHA, i.e. SFA, MUFA, LA, arachidonic acid and ALA, were also assessed by multivariate regression models for the $Z$-transformed scores of each fatty acid of interest. After constructing an unadjusted model, we adjusted for energy-adjusted EPA + DHA intake in a second model; finally, in a third model, we further considered as confounders those predictors with $P<0.05$ univariate associations (current smoking and physical activity). Statistical significance was defined as $P<0.05$. Analyses were performed using SPSS software, release 16.0 (SPSS, Inc., Chicago, IL, USA).

\section{Results}

The study population included 102 men and 96 women, aged 66 (SD 6) years. None of the study subjects had suffered prior CHD, but all of them were at high cardiovascular risk, as attested by the prevalence of overweight or obesity (92.9\%), family history of premature CHD (41.4\%), and/or treatment with antidiabetic, cholesterol-lowering and/or antihypertensive agents (85.4\%). Table 1 shows detailed information on

Table 1. Characteristics of the study population

(Mean values, standard deviations, number of subjects, percentages, ranges, medians and interquartile ranges, $n$ 198)

\begin{tabular}{|c|c|c|c|c|c|}
\hline Variables & Mean & SD & $n$ & $\%$ & Range \\
\hline Age (years) & 66 & 6 & & & $55-80$ \\
\hline Male & & & 102 & 51.5 & \\
\hline Current smoker & & & 31 & $15 \cdot 7$ & \\
\hline Former smoker & & & 65 & $32 \cdot 8$ & \\
\hline Dyslipidaemia & & & 149 & $75 \cdot 3$ & \\
\hline Hypertension & & & 171 & $86 \cdot 4$ & \\
\hline Type 2 diabetes mellitus & & & 73 & $36 \cdot 9$ & \\
\hline Hypolipidaemic drug treatment & & & 92 & $46 \cdot 5$ & \\
\hline Antihypertensive drug treatment & & & 131 & $66 \cdot 2$ & \\
\hline Antidiabetic drug treatment & & & 58 & $29 \cdot 3$ & \\
\hline BMI $\left(\mathrm{kg} / \mathrm{m}^{2}\right)$ & 29.2 & 3.2 & & & $21 \cdot 2-38.5$ \\
\hline Waist circumference (cm) & $100 \cdot 5$ & 8.5 & & & $76-127$ \\
\hline Systolic blood pressure (mmHg) & 148 & 20 & & & $96-196$ \\
\hline Diastolic blood pressure $(\mathrm{mmHg})$ & 81 & 10 & & & $55-111$ \\
\hline Leisure-time physical activity (MET-min*/d) & & & & & $0-1521$ \\
\hline Median & \multicolumn{2}{|c|}{484} & & & \\
\hline Interquartile ranges & \multicolumn{2}{|c|}{$316-676$} & & & \\
\hline \multicolumn{6}{|l|}{ Biochemistry values (mg/l) } \\
\hline Fasting glucose & 1240 & 410 & & & $660-3120$ \\
\hline Total cholesterol & 2110 & 420 & & & $1270-4810$ \\
\hline LDL-cholesterol ( $n$ 191) & 1330 & 330 & & & $350-2230$ \\
\hline HDL-cholesterol & 510 & 110 & & & $250-880$ \\
\hline TAG & & & & & $360-12680$ \\
\hline Median & \multicolumn{2}{|c|}{1120} & & & \\
\hline Interquartile ranges & \multicolumn{2}{|c|}{$840-1580$} & & & \\
\hline
\end{tabular}

${ }^{*}$ MET-min, minutes at a given metabolic equivalent level (units of energy expenditure in physical activity; 1 MET-min is roughly equivalent to $4.2 \mathrm{~kJ}(1 \mathrm{kcal}))$. 
Table 2. Proportions of the main fatty acids in whole blood cell membranes of the study population (Medians and interquartile ranges, $n$ 198)

\begin{tabular}{|c|c|c|}
\hline Fatty acids (\%) & Median & Interquartile range \\
\hline $12: 0$ & 0.06 & $0.03-0.16$ \\
\hline $14: 0$ & 0.77 & $0.25-1.03$ \\
\hline $16: 0$ & $22 \cdot 47$ & $21.57-23.44$ \\
\hline $18: 0$ & $14 \cdot 25$ & $11 \cdot 69-16.20$ \\
\hline $20: 0$ & 0.21 & $0.14-0.28$ \\
\hline $22: 0$ & 0.20 & $0.06-0.31$ \\
\hline$\Sigma S F A$ & 37.57 & $35.73-39.96$ \\
\hline $14: 1 n-5$ & 0.31 & $0.14-0.48$ \\
\hline $16: 1 n-7$ & 0.53 & $0.19-0.84$ \\
\hline cis,9-18: $1 n-9$ & $17 \cdot 64$ & $15 \cdot 87-19 \cdot 25$ \\
\hline $20: 1 n-9$ & 0.30 & $0.24-0.36$ \\
\hline $24: 1 n-9$ & 0.56 & $0.31-0.87$ \\
\hline ¿MUFA & 20.02 & $17 \cdot 9-21 \cdot 67$ \\
\hline $18: 2 n-6$ & 13.42 & $11 \cdot 7-15 \cdot 56$ \\
\hline $18: 3 n-6$ & 0.12 & $0.08-0.18$ \\
\hline $20: 3 n-6$ & 1.93 & $1.64-2.29$ \\
\hline $20: 4 n-6$ & $16 \cdot 52$ & $14 \cdot 62-18 \cdot 1$ \\
\hline$\sum n-6$ PUFA & 31.98 & $30.41-34.05$ \\
\hline $18: 3 n-3$ & 0.17 & $0.11-0.27$ \\
\hline $20: 5 n-3$ & 0.94 & $0.71-1.26$ \\
\hline $22: 5 n-3$ & 1.81 & $1.46-2.21$ \\
\hline $22: 6 n-3$ & 6.09 & $5 \cdot 16-6 \cdot 82$ \\
\hline$\Sigma n-3$ PUFA & $9 \cdot 21$ & $7.84-10 \cdot 42$ \\
\hline Omega-3 index & 7.05 & $6.08-8.05$ \\
\hline
\end{tabular}

clinical and anthropometric variables. The whole blood cell membrane fatty acid composition is presented in Table 2. The average omega-3 index was $7 \cdot 1 \%$ (Fig. 1), and was above $8 \%$ in $25.8 \%$ of the study group and below $4 \%$ in $3.5 \%$. Consumption of the fatty acids of interest is shown in Table 3. The intake of specific seafood items and their $\mathrm{EPA}+$ DHA content (Table 4) confirms the high consumption of total seafood of the study population. No significant differences were observed by sex or age regarding consumption of any of the seafood items (data not shown). None of the participants reported consumption of fish oil supplements. Attesting to the validity of the questionnaire used in the present study, Pearson's correlation coefficient values between the omega-3 index and both the total seafood intake and the calculated intake of EPA + DHA from seafood were 0.384 and 0.350 , respectively ( $P<0.000001$, both).

The univariate associations between the omega- 3 index and several clinical, anthropometric and dietary variables are

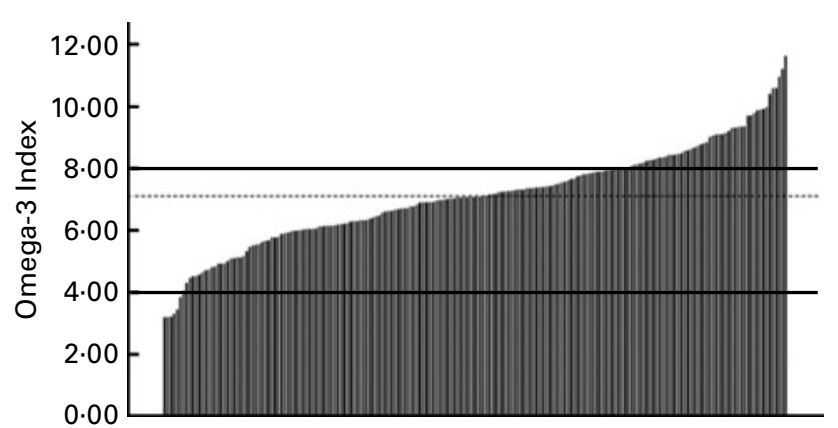

Fig. 1. Distribution of the percentage of whole blood cell EPA + DHA values (omega-3 index) in the study population ( $n$ 198). The omega- 3 index at 8 and $4 \%$ indicates proposed low- and high-risk horizons (-), respectively, while that at $7.1 \%$ is the population average $(\cdots)$.

shown in Table 5. The only factors significantly associated with the omega-3 index were serum TAG and smoking status (inversely), and leisure-time physical activity and $\mathrm{EPA}+\mathrm{DHA}$ intake (directly). Alcohol intake was not significantly associated with the omega-3 index. For every additional grams of EPA + DHA consumed per day, the omega- 3 index increased by 1.17 units. However, the most potent determinant of the omega- 3 index, the EPA + DHA intake, explained only $12 \%(P<0 \cdot 001)$ of the index's variability. The inclusion of another ten potential predictors to the model (sex, age, being a current smoker, treatment for diabetes, hypertension or dyslipidaemia, physical activity, BMI, and energy-adjusted dietary intakes of alcohol and total fat) increased the explanatory value of the index's variability to $19 \%$.

Table 6 shows the associations between the omega- 3 index and the intake of different fatty acids after adjustment for energy-adjusted intake of EPA + DHA. (Since other subjects' characteristics were not significant predictors in the multivariate model, they were not included in this model.) Consumption of EPA + DHA continued to be strongly related to the omega-3 index, while MUFA, LA, arachidonic acid and ALA were unrelated even in the unadjusted models. In contrast, the sum of dietary SFA showed a significant inverse association with the omega- 3 index in the unadjusted model. However, when the model was further adjusted for energyadjusted intake of EPA + DHA, current smoking and physical activity, the statistical significance of the association was blunted, although a trend for an inverse association was still present $(P=0 \cdot 095)$.

Table 3. Intake of fatty acids of interest in the study group

(Mean values, standard deviations and ranges)

\begin{tabular}{|c|c|c|c|c|c|c|}
\hline \multirow[b]{2}{*}{ Variables } & \multicolumn{3}{|c|}{ Intake (g/d) } & \multicolumn{3}{|c|}{$\begin{array}{l}\text { Intake as a percentage of } \\
\text { total energy }\end{array}$} \\
\hline & Mean & SD & Range & Mean & SD & Range \\
\hline Total fat & 105 & 27 & $48-225$ & $40 \cdot 2$ & $5 \cdot 2$ & $26 \cdot 0-54 \cdot 3$ \\
\hline SFA & 28 & 9 & $8-73$ & $10 \cdot 7$ & $2 \cdot 1$ & $4 \cdot 6-18 \cdot 1$ \\
\hline MUFA & 53 & 13 & $23-111$ & $20 \cdot 5$ & $3 \cdot 1$ & $12 \cdot 3-27 \cdot 8$ \\
\hline $18: 2 n-6$ & 12 & 4 & $4-29$ & 4.7 & $1 \cdot 3$ & $2 \cdot 5-9 \cdot 3$ \\
\hline $20: 4 n-6$ & 0.9 & 0.8 & $0.1-5.4$ & 0.4 & 0.3 & $0.0-2.4$ \\
\hline $18: 3 n-3$ & $1 \cdot 2$ & 0.4 & $0.4-3.4$ & 0.5 & $0 \cdot 1$ & $0.21-1 \cdot 1$ \\
\hline $20: 5 n-3+22: 6 n-3$ & 0.9 & 0.5 & $0.0-2 \cdot 1$ & 0.4 & 0.2 & $0.0-1.0$ \\
\hline
\end{tabular}


Table 4. Intake of seafood products and their associated EPA + DHA content

(Mean values, standard deviations and ranges)

\begin{tabular}{|c|c|c|c|c|c|}
\hline & \multicolumn{3}{|c|}{$\begin{array}{l}\text { Seafood products } \\
\qquad(\mathrm{g} / \mathrm{d})\end{array}$} & \multicolumn{2}{|c|}{$\begin{array}{r}E P A+D H A \\
\text { supplied (g/d) }\end{array}$} \\
\hline & Mean & SD & Range & Mean & SD \\
\hline Uncanned fatty fish & 25 & 21 & $0-102$ & 0.49 & 0.41 \\
\hline Lean fish & 44 & 25 & $0-118$ & 0.23 & 0.12 \\
\hline Smoked/salted fish & 3 & 4 & $0-26$ & 0.01 & 0.01 \\
\hline Molluscs & 4 & 4 & $0-26$ & 0.00 & 0.00 \\
\hline Shrimp, prawn and crayfish & 13 & 12 & $0-86$ & 0.03 & 0.04 \\
\hline $\begin{array}{l}\text { Octopus, baby } \\
\text { squid and squid }\end{array}$ & 17 & 19 & $0-86$ & 0.05 & 0.06 \\
\hline Fatty fish canned in oil & 8 & 8 & $0-32$ & $0 \cdot 10$ & 0.10 \\
\hline Fatty fish canned in water & 1 & 3 & $0-21$ & 0.01 & 0.02 \\
\hline Total seafood & 115 & 50 & $3-254$ & 0.92 & 0.49 \\
\hline
\end{tabular}

\section{Discussion}

In the present cross-sectional study, we searched for dietary, anthropometric and lifestyle determinants of the omega-3 index in a population at high risk of CHD living in Spain, the country with customarily high intakes of total fat, MUFA (supplied by olive oil), and EPA and DHA from seafood. Consistent with previous data, intake of EPA and DHA was the main predictor of the omega- 3 index, but explained only $12 \%$ of its variability. None of the other dietary fatty acids was related to the omega- 3 index.

Recognising that there are methodological differences in measuring the omega- 3 index, the mean value found in the present study group $(7 \cdot 1 \%)$ is noticeably higher than those described in Western populations, particularly in the USA, where it has been consistently reported to be about $5 \%{ }^{(6,7,9)}$. The cross-validation of our method against the method used in the original definition of the omega- 3 index (proposed by Harris and von Schacky) indicates that methodological differences do not explain the higher omega-3 index values seen in Spain. However, the value for the index reported here is still lower than those described in similar studies conducted in Japan ${ }^{(8)}$, Korea ${ }^{(23)}$ or Alaska (USA) ${ }^{(24)}$ The obvious explanation for the high omega- 3 index in the present study population is the high intake of EPA and DHA (mean $0.9 \mathrm{~g} / \mathrm{d}$ ), which is approximately the American Heart Association recommendation for subjects in secondary prevention $^{(3)}$. This, in turn, is due to the high consumption of seafood (mean $115 \mathrm{~g} / \mathrm{d}$ ), a value that concurs with those reported in recent surveys for different Spanish population groups $^{(14,15)}$. This high intake of EPA and DHA could in part explain the paradox of low rates of both incident CHD and cardiac death in Spain despite a high background prevalence of cardiovascular risk factors ${ }^{(25)}$. A similar paradox has been described in Japan ${ }^{(26)}$, the country leading in global fish consumption $^{(27)}$. In this regard, only $3.5 \%$ of the present study subjects showed an omega-3 index below $4 \%$, the level associated with an increased risk of fatal CHD, while $26 \cdot 1 \%$ displayed an index above $8 \%$, the level considered to be protective against CHD death ${ }^{(5)}$.

A unique feature of the present study was the examination of the potential effects on the omega-3 index of dietary fatty acids other than EPA + DHA. Of particular interest were LA and arachidonic acid because there is a controversy on whether increased consumption of these $n-6$ fatty acids

Table 5. Univariate associations of the omega-3 index with clinical, anthropometric and dietary variables*

\begin{tabular}{|c|c|c|c|c|}
\hline \multirow[b]{2}{*}{ Independent variables } & \multirow[b]{2}{*}{ Estimate } & \multicolumn{2}{|c|}{$95 \% \mathrm{Cl}$} & \multirow[b]{2}{*}{$P$} \\
\hline & & Lower & Upper & \\
\hline Age (10 years) & -0.215 & -0.584 & 0.154 & 0.253 \\
\hline Male, yes & -0.247 & -0.694 & 0.200 & 0.227 \\
\hline Current smoker, yes & -0.653 & -1.263 & -0.044 & 0.036 \\
\hline Former smoker, yes & 0.041 & -0.436 & 0.518 & 0.864 \\
\hline Dyslipidaemia, yes & -0.099 & -0.618 & 0.420 & 0.708 \\
\hline Hypertension, yes & 0.547 & -0.102 & $1 \cdot 195$ & 0.098 \\
\hline Type 2 diabetes mellitus, yes & 0.033 & -0.431 & 0.498 & 0.888 \\
\hline Hypolipidaemic drug treatment, yes & 0.051 & -0.398 & 0.500 & 0.824 \\
\hline Antihypertensive drug treatment, yes & 0.280 & -0.192 & 0.752 & 0.243 \\
\hline Antidiabetic drug treatment, yes & 0.223 & -0.269 & 0.714 & 0.373 \\
\hline BMI $\left(3.12 \mathrm{~kg} / \mathrm{m}^{2}\right) \dagger$ & -0.026 & -0.250 & 0.199 & 0.821 \\
\hline Waist circumference $(8.5 \mathrm{~cm}) \dagger$ & -0.175 & -0.398 & 0.048 & 0.124 \\
\hline Systolic blood pressure $(19.9 \mathrm{mmHg}) \dagger$ & 0.005 & -0.221 & 0.231 & 0.966 \\
\hline Diastolic blood pressure $(10.1 \mathrm{mmHg}) \dagger$ & 0.048 & -0.177 & 0.274 & 0.674 \\
\hline Leisure-time physical activity (293.7 MET-min $\ddagger / d) \dagger$ & 0.271 & 0.048 & 0.494 & 0.018 \\
\hline Fasting glucose $(408 \mathrm{mg} / \mathrm{l}) \dagger$ & -0.064 & -0.290 & 0.162 & 0.577 \\
\hline Total cholesterol $(418 \mathrm{mg} / \mathrm{l}) \dagger$ & -0.193 & -0.418 & 0.032 & 0.092 \\
\hline LDL-cholesterol $(321 \mathrm{mg} / \mathrm{l}) \dagger(n$ 191) & 0.012 & -0.213 & 0.238 & 0.913 \\
\hline HDL-cholesterol $(112 \mathrm{mg} / \mathrm{l}) \dagger$ & -0.077 & -0.304 & $0 \cdot 150$ & 0.505 \\
\hline TAG $(1363 \mathrm{mg} / \mathrm{l}) \dagger$ & -0.299 & -0.522 & -0.077 & 0.009 \\
\hline Energy-adjusted alcohol intake (g/d) & 0.010 & -0.005 & 0.026 & $0 \cdot 190$ \\
\hline Energy-adjusted total fat intake $(\mathrm{g} / \mathrm{d})$ & -0.001 & -0.009 & 0.008 & 0.839 \\
\hline Energy-adjusted intake of EPA + DHA $(\mathrm{g} / \mathrm{d})$ & $1 \cdot 178$ & 0.742 & $1 \cdot 613$ & $<0.001$ \\
\hline
\end{tabular}


Table 6. Multivariate associations between energy-adjusted fatty acid intake and the omega-3 index in the study population $(n 198)^{\star}$

\begin{tabular}{|c|c|c|c|c|c|c|c|c|c|c|c|c|c|}
\hline \multirow[b]{3}{*}{ Fatty acids } & \multirow[b]{3}{*}{ SD } & \multicolumn{4}{|c|}{ Model 1† } & \multicolumn{4}{|c|}{ Model 2‡ } & \multicolumn{4}{|c|}{ Model 3§ } \\
\hline & & \multirow[b]{2}{*}{ Estimate } & \multicolumn{2}{|c|}{$95 \% \mathrm{Cl}$} & \multirow[b]{2}{*}{$P$} & \multirow[b]{2}{*}{ Estimate } & \multicolumn{2}{|c|}{$95 \% \mathrm{Cl}$} & \multirow[b]{2}{*}{$P$} & \multirow[b]{2}{*}{ Estimate } & \multicolumn{2}{|c|}{$95 \% \mathrm{Cl}$} & \multirow[b]{2}{*}{$P$} \\
\hline & & & Lower & Upper & & & Lower & Upper & & & Lower & Upper & \\
\hline$E P A+D H A$ & 0.49 & 0.57 & 0.36 & 0.78 & $<0.001$ & - & - & - & - & 0.52 & 0.30 & 0.73 & $<0.001$ \\
\hline SFA & 9.22 & -0.25 & -0.47 & -0.03 & 0.026 & -0.20 & -0.41 & 0.01 & 0.066 & -0.18 & -0.39 & 0.03 & 0.095 \\
\hline MUFA & 13.00 & 0.06 & -0.16 & 0.29 & 0.582 & 0.09 & -0.12 & 0.30 & 0.405 & 0.09 & -0.12 & 0.31 & 0.376 \\
\hline LA & 4.48 & 0.02 & -0.21 & 0.24 & 0.873 & 0.07 & -0.14 & 0.28 & 0.513 & 0.07 & -0.14 & 0.28 & 0.518 \\
\hline $\mathrm{AA}$ & 0.82 & -0.01 & -0.24 & 0.21 & 0.903 & -0.38 & -0.25 & -0.17 & 0.719 & -0.02 & -0.24 & 0.19 & 0.827 \\
\hline ALA & 0.43 & 0.07 & -0.16 & 0.29 & 0.568 & 0.02 & -0.19 & 0.23 & 0.833 & 0.04 & -0.17 & 0.26 & 0.680 \\
\hline
\end{tabular}

LA, linoleic acid; AA, arachidonic acid; ALA, $\alpha$-linolenic acid.

* Data are presented for a 1 standard deviation unit increase in the fatty acid of interest, examined by multiple linear regression analyses.

$\dagger$ Unadjusted.

$\ddagger$ Adjusted for EPA + DHA intake (energy-adjusted).

$\S$ Further adjustment for current smoking and total physical activity (increase of 293.7 MET-min/d). MET-min, minutes at a given metabolic equivalent level (units of energy expenditure in physical activity; $1 \mathrm{MET}$-min is roughly equivalent to $4.2 \mathrm{~kJ}(1 \mathrm{kcal})$ ).

would decrease the proportions of EPA and DHA in membranes ${ }^{(11-13)}$. ALA intake was of interest since it is the precursor of EPA and $\mathrm{DHA}^{(2)}$. We found that the intake of none of these fatty acids was related to the omega- 3 index. It is possible that ALA might have had more influence on the omega-3 index had the EPA intake not been so high, since EPA can inhibit the activity of $\delta-5$ and $\delta$ - 6 desaturases, which are required for the conversion of ALA into longerchain derivatives ${ }^{(2)}$. On the other hand, the relatively low intake of LA (mean $12 \mathrm{~g} / \mathrm{d}$, owing to the low consumption of seed oils, margarines and shortenings in Spain) would be expected to enhance the conversion of ALA to EPA by reduced competition for the desaturases.

There were significant associations of current smoking and physical activity with the omega- 3 index, findings that concur with data from prior studies ${ }^{(8)}$. The inverse relationship between the omega- 3 index and serum TAG has also been reported previously ${ }^{(7)}$, and probably reflects the well-known effects of EPA and DHA on this serum lipid fraction ${ }^{(28)}$. Finally, in contrast to the findings of the IMMIDIET study ${ }^{(22)}$, the omega-3 index was unrelated to alcohol intake, even though average consumption was similar in the two studies. The higher EPA + DHA intakes in Spain compared with those in the countries included in the IMMIDIET study suggest that alcohol intake may only affect the omega-3 index in the context of low $n-3$ intakes.

The present study has limitations. First, it was a singlecentre study with a relatively small sample size. Second, given the cross-sectional design of the study, temporal relationships cannot be established, and we cannot exclude the possibility of residual confounding. Strengths of the study included the use of a validated FFQ that is comprehensive regarding seafood intake, the validation of the fatty acid analysis method against a reference laboratory and the specific focus on individuals at increased risk for cardiac death. To our knowledge, this is the first examination of the omega-3 index in a Spanish cohort

In conclusion, in a population at high risk of $\mathrm{CHD}$ with high intakes of total fat, MUFA and EPA + DHA, the omega-3 index was primarily predicted by the intake of EPA + DHA, not by known demographic, metabolic or other lifestyle factors.
Further research is needed to more clearly define the environmental and genetic determinants of this emerging risk factor for CHD.

\section{Acknowledgements}

Emili Corbella provided expert assistance with statistical analyses. CIBERobn is an initiative of ISCIII, Spain. W. S. H. is a scientific advisor to companies with interests in fatty acids including Monsanto, Unilever, Omthera and GlaxoSmithKline, and has been a speaker for the latter. In addition, he is the owner of OmegaQuant, LLC, a company that offers blood $n$-3 fatty testing. E. R. is a scientific advisor to Ferrer International, the company that commercialises Omacor (a brand of $n-3$ fatty acid supplements) in Spain. None of the other authors has any potential conflicts to disclose. The present study was supported by grants FIS PI06/0365 from the Spanish Health Ministry, Centro Nacional de Investigaciones Cardiovasculares 2008 and Fundació Privada Catalana de Nutrició i Lípids, Barcelona, Spain. A. S.-V. was supported by post-doctoral contract FIS CD07/0083. The author's responsibilities were as follows: A. S.-V., W. S. H. and E. R. designed the study; A. S.-V. and M. C. determined the fatty acid composition of whole blood cell membranes; A. S.-V., W. S. H. and E. R. drafted the manuscript; all other authors substantially contributed to the analysis and/or interpretation of the data and revised the manuscript critically for important intellectual content.

\section{References}

1. Lavie CJ, Milani RV, Mehra MR, et al. (2009) Omega-3 polyunsaturated fatty acids and cardiovascular diseases. $J \mathrm{Am}$ Coll Cardiol 54, 585-594.

2. Brenna JT, Salem N Jr, Sinclair AJ, et al. (2009) Alpha-linolenic acid supplementation and conversion to $n-3$ long-chain polyunsaturated fatty acids in humans. Prostaglandins Leukot Essent Fatty Acids 80, 85-91.

3. Lucas M, Asselin G, Plourde M, et al. (2010) n-3 Fatty acid intake from marine food products among Quebecers: comparison to worldwide recommendations. Public Health Nutr 13, 63-70. 
4. Baylin A \& Campos H (2006) The use of fatty acid biomarkers to reflect dietary intake. Curr Opin Lipidol 17, $22-27$.

5. Harris WS (2009) The omega-3 index: from biomarker to risk marker to risk factor. Curr Atheroscler Rep 11, 411-417.

6. Sands SA, Reid KJ, Windsor SL, et al. (2005) The impact of age, body mass index, and fish intake on the EPA and DHA content of human erythrocytes. Lipids 40, 343-347.

7. Block RC, Harris WS \& Pottala JV (2008) Determinants of blood cell omega-3 fatty acid content. Open Biomark J 1, $1-6$.

8. Itomura M, Fujioka S, Hamazaki K, et al. (2008) Factors influencing EPA + DHA levels in red blood cells in Japan. In vivo 22, 131-135.

9. Cohen BE, Garg SK, Ali S, et al. (2008) Red blood cell docosahexaenoic acid and eicosapentaenoic acid concentrations are positively associated with socioeconomic status in patients with established coronary artery disease: data from the Heart and Soul Study. J Nutr 138, 1135-1140.

10. Whelan J (2008) The health implications of changing linoleic acid intakes. Prostaglandins Leukot Essent Fatty Acids 79, $165-167$.

11. Kris-Etherton P, Fleming J \& Harris WS (2010) The debate about $n-6$ polyunsaturated fatty acid recommendations for cardiovascular health. J Am Diet Assoc 110, 201-204.

12. Harris WS, Mozaffarian D, Rimm E, et al. (2009) Omega-6 fatty acids and risk for cardiovascular disease: a science advisory from the American Heart Association Nutrition Subcommittee of the Council on Nutrition, Physical Activity, and Metabolism; Council on Cardiovascular Nursing; and Council on Epidemiology and Prevention. Circulation 119, 902-907.

13. Ramsden C,Hibbeln J, Lands B, et al. (2009) Letters to the Editor and writing group response regarding "Omega- 6 fatty acids and risk for cardiovascular disease" - Letters (CIRCULATIONAHA/2009/865667; CIRCULATIONAHA/2009/ 866566; CIRCULATIONAHA/2009/866434) submitted by Ramsden C, Hibbeln J, Lands B \& Tribole E. American Heart Association web site. http://americanheart.org/ downloadable/heart/12566483387500mega6letterswresp.pdf (accessed 4 January 2010).

14. Welch AA, Lund E, Amiano P, et al. (2002) Variability of fish consumption within the 10 European countries participating in the European Investigation into Cancer and Nutrition (EPIC) study. Public Health Nutr 5, 1273-1285.

15. Garcia-Closas R, Berenguer \& González CA (2006) Changes in food supply in Mediterranean countries from 1961 to 2001. Public Health Nutr 9, 53-60.
16. Estruch R, Martínez-González MA, Corella D, et al. (2006) Effects of a Mediterranean-style diet on cardiovascular risk factors: a randomized trial. Ann Intern Med 145, 1-11.

17. Elosua R, Marrugat J, Molina L, et al. (1994) Validation of the Minnesota leisure time physical activity questionnaire in Spanish men. Am J Epidemiol 139, 1197-1209.

18. Elosua R, Garcia M, Aguilar A, et al. (2000) Validation of the Minnesota leisure time physical activity questionnaire in Spanish women. Med Sci Sports Exerc 32, 1431-1437.

19. Fernández-Ballart JD, Piñol JL, Zazpe I, et al. (2010) Relative validity of a semi-quantitative food-frequency questionnaire in an elderly Mediterranean population of Spain. Br J Nutr 103, 1808-1816.

20. Moreiras O, Carbajal A, Cabrera L, et al. (2005) Tablas de Composición de Alimentos (Food Composition Tables). Madrid: Ediciones Pirámide, SA.

21. Willett WC, Howe GR \& Kushi LH (1997) Adjustment for total energy intake in epidemiologic studies. Am J Clin Nutr $\mathbf{6 5}$, S1220-S1228.

22. di Giuseppe R, de Lorgeril M, Salen P, et al. (2009) Alcohol consumption and $n-3$ polyunsaturated fatty acids in healthy men and women from 3 European populations. Am J Clin Nutr 89, 354-362.

23. Park Y, Park S, Yi H, et al. (2009) Low level of $n-3$ polyunsaturated fatty acids in erythrocytes is a risk factor for both acute ischemic and hemorrhagic stroke in Koreans. Nutr Res 29, 825-830.

24. Ebbesson SO, Devereux RB, Cole S, et al. (2010) Heart rate is associated with red blood cell fatty acid concentration: the Genetics of Coronary Artery Disease in Alaska Natives (GOCADAN) study. Am Heart J 159, 1020-1025.

25. Marrugat J, D'Agostino R, Sullivan L, et al. (2003) An adaptation of the Framingham coronary heart disease risk function to European Mediterranean areas. $J$ Epidemiol Community Health 57, 634-638.

26. Ueshima H (2007) Explanation for the Japanese paradox: prevention of increase in coronary heart disease and reduction in stroke. J Atheroscler Thromb 14, 278-286.

27. Iso H, Kobayashi M, Ishihara J, et al. (2006) Intake of fish and $n 3$ fatty acids and risk of coronary heart disease among Japanese: the Japan Public Health Center-Based (JPHC) Study Cohort I. Circulation 113, 195-202.

28. Harris WS \& Bulchandani D (2006) Why do omega-3 fatty acids lower serum triglycerides? Curr Opin Lipidol 17, 387-393. 